\title{
Safeguarding clinical trials
}

\section{Efforts are underway to modernize clinical trial standards and normalize regulations to facilitate international collaboration. But as the European Union's Clinical Trials Directive shows, a one-size-fits-all regulatory strategy may be easier to conceive than to implement.}

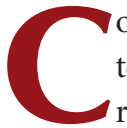
onducting clinical trials has never been easy, but when testing a new drug or therapy can risk lives, it stands to reason that the public should demand strict oversight. Yet the public is equally impatient for better disease treatments, and increased regulation has the potential to slow the delivery of new cures. Is there a way to streamline the approval process while still ensuring participants' safety?

In 2004, the European Union (EU) issued the Clinical Trials Directive intended to do just that: simplify the regulation of clinical trials in member nations while increasing protection of patients. By establishing clear guidelines for the use of Good Clinical Practice and procedures for coordinating international clinical trials, the directive further aimed to facilitate drug approval and the marketing of medicinal products in the EU. Yet even prior to its implementation, concerns were voiced in academic circles that the directive might add to the complexity of clinical trials and ultimately impede licensing of new therapies.

A major problem with the EU directive is that, unlike a law, implementation of its guidelines can differ among the member nations, counteracting the intended goal of trial normalization and complicating international collaboration.

Moreover, in establishing standards for clinical trials, the directive doesn't substantially differentiate between industry-sponsored and academic trials. In both cases, a single sponsor is now required to take responsibility for the "initiation, management and/or financing of a clinical trial." A single sponsor may be the norm for industry trials. But academic trials are co-run by foundations, charities, medical centers and universities and are not accustomed to a single entity being held fully accountable. Finding a sponsor that will shoulder the entire legal responsibility is now a significant hurdle.

The directive also imposes new stipulations on the manufacture and labeling of medicinal products used in trials and more stringent safety reporting and trial monitoring. Adherence to the new standards requires increased time, infrastructure and possibly staff, thereby raising the costs of trials. Such new financial constraints may ultimately prohibit the testing of innovative therapies by academic researchers, leading to a decrease in the number of clinical trials and eventually to fewer treatment options.
Now, two years into the directive's launch, some of these fears are proving justified (p. 110). A recent study of eight Clinical Trials Units in the UK confirmed that the directive dramatically augments the regulatory and financial burden for non-commercial clinical trials and reduces the incentive to engage in international trials because of the variable interpretation of the directive across EU states (Eur. J. Cancer 43, 8-13; 2007). For non-commercial cancer trials, the study estimated that the directive has doubled the cost, increased the complexity of paperwork and created impediments to finding a trial sponsor. Failure to obtain a sponsor prevented three units from engaging in any new trials since the directive's formal implementation in May 2004.

The Clinical Trials Unit's investigators conceded that fulfilling the documentation requirements would become easier with time, but concluded that the EU directive had failed in its intended attempts to harmonize and simplify the regulation of clinical trials.

In the US, the Food and Drug Administration (FDA) is also updating the regulations governing the management and monitoring of clinical trials to keep in step with advances in electronic data storage and the trend to large, decentralized, multi-site trials. In June 2006, the US FDA announced the launch of The Human Subject Protection and Bioresearch Monitoring Initiative, which aims to increase the oversight of trials and the protection of participants and to improve the integrity of trial data. Thus far, the FDA has published several informational reports on operating, monitoring and reporting on clinical trials in order to eventually draft new rules.

The efforts in the EU and the US to simplify the regulatory burden of clinical trials, improve patient safety and speed approval of new therapies are certainly laudable. But that combination of factors may be working at cross-purposes, and the worst-case outcome may be fewer clinical trials, a drop in international collaboration, increased outsourcing of trials and a loss of diversity in new treatments. If the efforts are to succeed, a flexible approach is essential to revise the regulations if their impact proves detrimental-and preferably in less time that the duration of the average clinical trial. 